\title{
Translational Nursing Science: Implementation of a Patient Fall Reduction Strategy in the Emergency Department at a Community-Based Medical Center
}

\author{
William G Zic* \\ Assistant Professor, Saint Xavier University, USA
}

\begin{abstract}
Current literature suggests that a multitude of fall prevention methods, practices and tools exist and are widely available for organizations to choose from. Although much patient fall reduction information is easily accessible, very few fall prevention tools are Emergency Department (ED) specific.

Goal: The main goal of this project was to reduce or eliminate patient falls in the ED by implementing a customized set of Agency for Healthcare Research and Quality (AHRQ) patient fall reduction guidelines.

PICOT: For adult patients age 18 and over in the Emergency Department at a community medical center, how can the implementation of AHRQ Patient Fall Reduction Guidelines, compared to current practice, effect patient fall outcomes, over a three-month time period?

Method: Falls in the ED were calculated using a patient fall calculation method (number of patient falls x 1000 / number of patients seen in the ED). The KINDER 1 Fall Risk Assessment Tool was used to quickly identify patients at risk for falls. During project implementation, one patient experienced a fall. The number of patients who met fall risk assessment criteria was 328 out of 3,323 adult patients. Patients age 17 or younger were omitted from project data collection and participation. When a patient was identified as a fall risk, additional patient fall interventions were implemented.
\end{abstract}

Results/Outcomes: Prior to implementation of AHRQ guidelines, the hospital's previous annual ED fall rate average was $1.06 \%$ The fall rate percentage at the completion of this project was $0.30 \%$ over a three-month time frame from January 2019 through March 2019. Fall rate comparison showed, with a 95\% confidence interval, no major change in fall rate during the length of this project. However, there was a downward trend in patient fall rate that was well established.

Nursing Implication: Additional research is needed that will compare quarterly fall rates over an extended period of time in order to better understand the underlying causes that contribute to patient falls and improved patient safety in the ED across various health systems.

KEYWORDS: Fall prevention; KINDER1; Interventions; AHRQ tools

ABBREVIATIONS: CMS: Center for Medicare and Medicaid Services; ED: Emergency Department; HAC: Hospital Acquired Conditions; AHRQ: Agency for Healthcare Research and Quality; HFRM: Hendrich II Fall Risk Model; MFS: Morse Falls Scale

Quick Response Code:

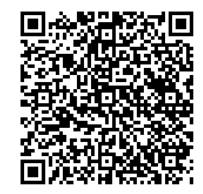

Address for correspondence: William G Zic, Assistant Professor, Saint Xavier University, USA

Received: February 04, 2020 Published: March 04, 2020

How to cite this article: William GZ. Translational Nursing Science: Implementation of a Patient Fall Reduction Strategy in the Emergency Department at a Community-Based Medical Center. 2020 - 2(2) OAJBS.ID.000148. DOI: 10.38125/OAJBS.000148 


\section{EMERGENCY DEPARTMENT FALL REDUCTION}

Patient falls continue to be an extremely challenging conundrum for healthcare organizations. Many falls result in direct injury, which can lead to prolonged hospital stay, unnecessary hospital expenditures and negative patient outcomes. McCarty [1] reported that $30 \%-50 \%$ of patient falls result in injury and add an estimated $\$ 31$ billion dollars in extra medical costs each year. Because patient falls should either be reduced or avoided altogether, the Center for Medicare and Medicaid services (CMS) proclaimed several years ago, that it would no longer financially reimburse hospitals for additional treatment costs related to patient falls.

Even though there is a large body of evidence regarding patient fall prevention in acute care inpatient settings, very little patient fall research has been performed in the outpatient setting, such as the Emergency Department (ED). Because patient falls continue to be a high-priority healthcare concern, a comprehensive adult patient fall prevention strategy was implemented in the ED at a community-based medical center.

\section{Literature Review}

According to the Joint Commission [2] the first steps to successful fall prevention involve the proper assessment and education of patients at risk for falling within the first 24 hours of hospital admission. France [3] discussed how hospital acquired conditions (HAC's), such as patient falls, have been reduced in recent years. However, the Agency for Healthcare Research and Quality (AHRQ) [4] estimated that hospital patient falls rates have remained steady and included patient falls in their "Never Event" criteria list. In other words, with the appropriate organizational fall prevention strategy in place, patient falls should either be preventable or simply should not occur.

Pua $[5,6]$ concluded that older adults have an increased risk for falls, hospital readmission, functional decline and mortality. In a study by Goldberg, McCreedy [7] the authors reported that many patients fall among adults in the emergency department ED lead to complicated and expensive care delivery. Surprisingly, the authors also found that medical professionals in the ED may not properly evaluate all patients who may be at risk. Townsend [8] conducted a four-month study within the emergency department at a rural hospital. This study compared the use of the KINDER 1 Fall Risk Assessment Tool (KINDER 1) to the Hendrich II Fall Risk Model (HFRM) and Morse Falls Scale (MFS). Both the MFS and the HFRM have been utilized as inpatient fall risk tools, whereas the KINDER 1 is ED specific. The results of this study proved that the KINDER 1 assisted to reduce patient falls by almost $20 \%$ more than either the MFS or HFRM-thereby making it a better patient fall reduction tool for the ED. The authors further concluded that because of its initial use at the point of patient entry (triage), the KINDER 1 also proved to more rapidly identify those patients at risk for falls. This, in turn, fostered staff communication between the triage and bedside areas creating a greater overall patient fall awareness amongst all ED staff.

Alexander [9] explained that little evidence-based literature is available which has examined ED specific patient fall reduction strategies. Because of this, the authors conducted a study that retrospectively implemented the KINDER 1 Fall Risk Assessment Tool to an existing ED patient fall reduction strategy. The results proved that when the KINDER 1 was applied, it helped to further identify and capture an additional $49 \%$ of fall risk patients, which helped to identify close to $100 \%$ of all ED patients as high fall risksbefore a fall. Although the KINDER 1 Fall Risk Assessment Tool has been utilized for less than a decade in the healthcare industry, it has proved to reliably validate several recent ED patients fall reduction studies. Therefore, this patient fall risk assessment tool was selected as the tool of choice to assist in identifying those patients at risk for falls in the ED for this project.

\section{Pre-Implementation Plan}

\section{PICOT Question}

The following PICOT question served as the basis for the ED fall reduction project: For adult patients visiting the emergency department, does the implementation of the Agency for Healthcare Research and Quality Fall Prevention Guidelines, compared to current practice, impact patient fall rates over three months?

\section{Instrumentation}

The Agency for Healthcare Quality and Research (AHRQ) recommends the use of several tools to create a multifactorial approach toward a fall prevention strategy. This project followed AHRQ guidelines and involved several customized AHRQ patient fall reduction tools. The AHRQ tools utilized were: KINDER 1 Fall Risk Assessment Tool, Fall Rate Calculation Formula, Interdisciplinary Team Tool (list of staff), Action Plan (staff responsibilities), Post Fall Assessment, Patient Fall Pathway Guide, Schedule Rounding Protocol (standard patient fall interventions), Patient and Family Education, Assessing Care Processes (audit tool), and a Sustainability Tool. However, only three of these instruments were useful for measurement purposes and statistical analysis. The instruments used for statistical analysis in this project were: The KINDER 1 Fall Risk Assessment Tool, Accessing Care Processes Tool (audit tool) and a standardized ED Patient Fall Rate Calculation Tool.

\section{Population}

The primary participants of this project included 328 adult patients over age 18 who presented to the ED and met any one of the fall risk inclusion criteria found on the KINDER 1 Fall Risk Assessment Tool.

\section{Comparison}

The community based medical center had previously utilized the Hendrich II Fall Assessment in the Emergency Department. However, in 2017, ED leadership elected to omit use of the Hendrich II because it was lengthy to complete, was not specific to the ED and contained patient fall risk information that was better suited for inpatient nursing units. As a result, the ED experienced a high number of adult patient falls, which was directly attributed to the lack of use of a patient fall prevention strategy.

\section{Sample and setting}

The project site was a small, but very busy 250-bed community medical center located in Northern Illinois. The facility routinely provides ED service to approximately 15,000 to 20,000 people per year from the surrounding community and beyond. Physicians, Advanced Practice Nurses, Staff Nurses, ED Technicians, Unit Clerks and Security Guards staff the ED at the project site and in 2018, the average number of patients seen per day in the ED was 63.

\section{Implementation of the Fall Reduction Strategy}

Beginning in the first week of January 2019, all emergency nurses and staff on all shifts were instructed on the use of all Patient Fall Reduction Tools utilized in this project. The triage or bedside nurse in the case of direct ED admissions, implemented the KINDER 1 Tool on all patients age 18 and over. If any adult patients 
met anyone of the KINDER 1 criterion, a patient was considered at risk for falls. However, staff was also encouraged to continue to answer all remaining questions on the KINDER 1 Tool, which included a free text section for additional comments. For example, additional documentation by the ED nurse could include, bowel or bladder information and hypotension (Figure 1).

\begin{tabular}{|l|l|l|}
\hline \multicolumn{1}{|c|}{ RISK } & YES & NO \\
\hline Present to ED because of fall & & \\
\hline Age>70 & & \\
\hline $\begin{array}{l}\text { Altered Mental Status } \\
\text { Intoxicated with Alcohol or } \\
\text { Substance Confusion }\end{array}$ & & \\
\hline $\begin{array}{l}\text { Impaired Mobility: } \\
\text { ambulates or transfers with } \\
\text { Ambulates with unsteady gait } \\
\text { and no assistance } \\
\text { Unable to ambulate or transfer }\end{array}$ & & \\
\hline Nursing Judgment (free text) & & \\
\hline
\end{tabular}

Figure 1: KINDER 1 fall risk assessment tool.

*Adapted with permission from terry kinsley, 745 Burnside Ave, East Hartford, СT 0610.

Once a patient was identified as a fall risk, the triage or bedside nurse notified a member of nursing leadership, such as either the charge nurse or nurse manager depending on availability. The nurse leader then immediately called an ad-hoc all staff huddle to discuss the activation of additional patient fall intervention processes. Additional patient falls risk intervention processes included the use of a Patient Fall Pathway Guide, which assisted to illustrate further staff responsibilities, and the use of a Patient and Family Education Document. The Patient and Family Education Tool assisted the nurse to communicate basic patient fall reduction information to patients and their respective family members. As an example, a patient or family member was reminded to activate the call light when needing any assistance and patients were reminded to never attempt getting out of bed without staff assistance. Patient and Family Education information was omitted when patients had an altered mental status, such as confusion or delirium, or in such cases when patients did not have an accompanying family member (Figure 2).

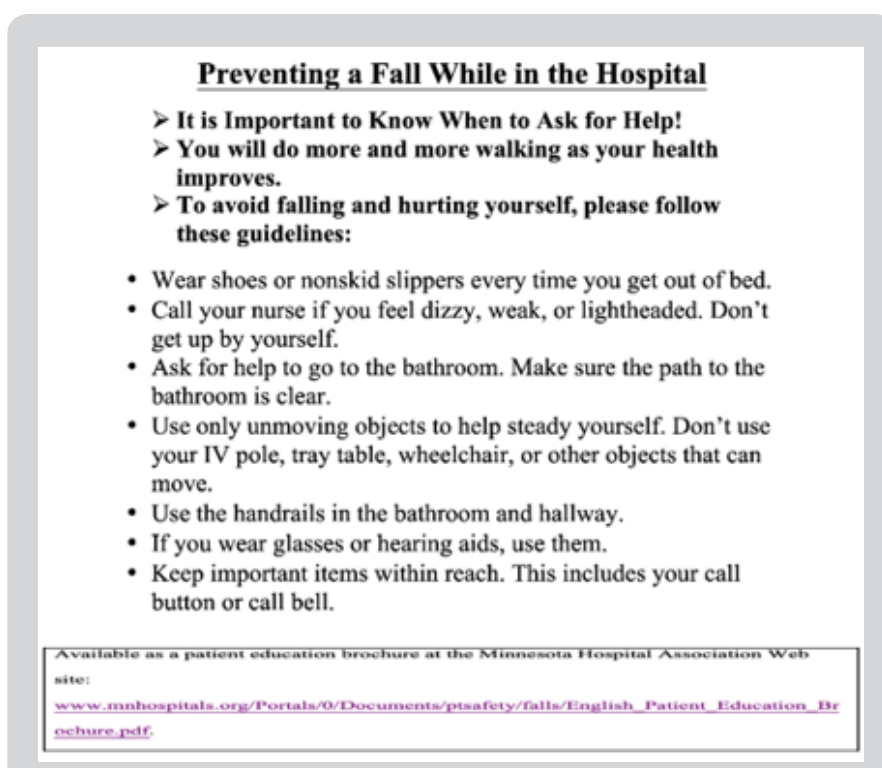

Figure 2: Patient and family education tool.

Another tool, the Schedule Rounding Protocol Tool was also activated when a patient was identified as a fall risk. This protocol was exercised in similar fashion to hourly patient rounding documentation and contained a set of standard hospital fall prevention interventions. For instance, this checklist document reminded staff to: move fall risk patients close to the nurses station when able, place patient beds into the lowest position with side rails up, provide patients with non-skid footwear, place call lights and other items within patient reach, provide adequate room lighting, apply yellow bracelets to a patients limb, place a patient into a yellow gown, hang a yellow star outside a fall risk patients room and remind patients and family members of fall prevention information. Screening and monitoring of patients at risk for falls continued in this fashion through March 2019 (Figure 3).

\begin{tabular}{|c|c|}
\hline 1 & Assess patient pain levels using a pain-assessment scale. \\
\hline 2 & Offer pain medicine when the dose is due. \\
\hline 3 & Offer toileting assistance. \\
\hline 4 & Make sure bedrails are up* \\
\hline 5 & Check that the bed is in locked position. ${ }^{*}$ \\
\hline 6 & Place bed in low position when patient is resting. * \\
\hline 7 & $\begin{array}{l}\text { Make sure the call light, telephone, bedside table, garbage can and patient belongings } \\
\text { (when applicable) are within the patient's reach and patient can demonstrate use. }{ }^{*}\end{array}$ \\
\hline 8 & Provide adequate room lighting. ${ }^{*}$ \\
\hline 9 & Apply yellow star outside patient room. * \\
\hline 10 & Perform hourly patient rounding per hospital policy. ${ }^{*}$ \\
\hline 11 & Educate patients and family members on fall reduction techniques. ${ }^{*}$ \\
\hline 12 & Check that patient has non-slip footwear and correct (yellow) armband applied. * \\
\hline 13 & $\begin{array}{l}\text { Prior to leaving the room, ask, "Is there anything I can do for you before I leave? I have time } \\
\text { while I am here in the room." }\end{array}$ \\
\hline 14 & $\begin{array}{l}\text { Remind patient that a member of the nursing staff (use names on white board) will be back } \\
\text { in the room in an hour to round again. }\end{array}$ \\
\hline
\end{tabular}

Figure 3: Schedule rounding protocol tool (hospital patient falls precautions).

*This tool is a component of the AHRQ toolkit and is in the public domain. 
Assessing Care Processes Tools (audit tools) were also applied each week. These documents were very useful to assure KINDER 1 tool completion, Schedule Rounding Protocol implementation, Patient Family and Education implementation and Post Fall Assessment completion when appropriate. Additionally, the Assessing Care Processes Tool involved a series of questions requiring a yes, no or in certain cases, a not applicable (N/A) answer. For example, tasks were completed (Yes), not completed (No) or were not applicable (N/A) given patient type or situation.
Not applicable answers either related to a patient's inability to receive education, lack of family participation in patient care or a patient did not experience a fall. Direct observation and feedback were regularly provided by the project leader and members of ED leadership in order to drive high compliance percentages in each category. If staff failed to perform any task related to any question on any auditable tool, simple redirection occurred. This redirection allowed for immediate correction, completion and documentation of AHRQ Patient Fall Reduction Tools (Figure 4).

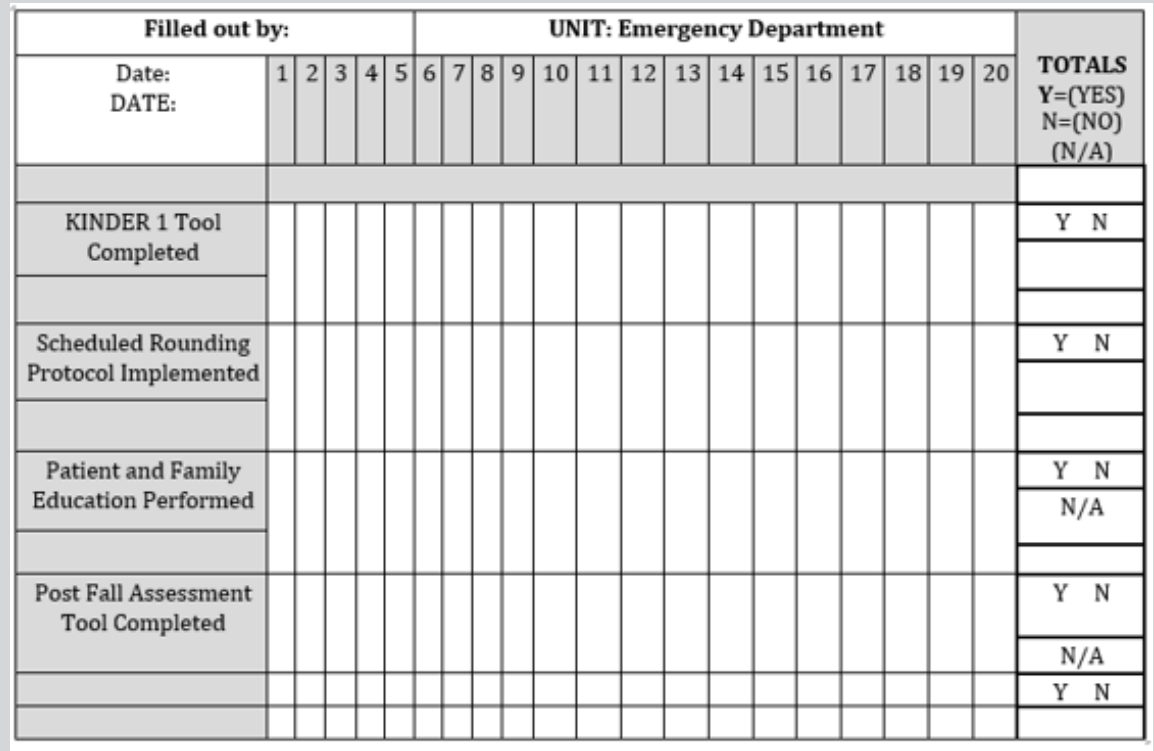

Figure 4: Assessing care processes tool (audit form).

*Adapted with permission from: royal college of physicians implementing fall safe: care bundles to reduce patient falls. London, UK: Royal College of Physicians; 2012. Available at: www.rcplondon.ac.uk/resources/falls-preventionresources

*This tool is a component of the AHRQ toolkit and is in the public domain.

\section{DISCUSSION}

Statistical analysis that compared fall rates was analyzed by Medcalc(C) statistical software. Medcalc( $)$ was selected as the software for this project because it is widely used by various healthcare professionals, quickly and effectively measures raw data scores and reliably analyzes and compares basic epidemiological features of a given population. For comparison differences in fall rates, standardized rate difference calculations, techniques and applications were performed based upon the Khurshid method of statistics in epidemiology. The Khurshid method of statistics utilizes powerful, yet common statistical formulas to analyze data for simple comparison.

Patient fall rate calculations involved simple multiplication and a numerator over denominator approach to problem solving. These rates were calculated using the following formula: (number of patient falls x 1000 / number of patients seen in the ED). The results were compared to historical quarterly percentages using a 95\% confidence interval (CI). Adults age 18 and over were screened for project participation using the KINDER 1 Fall Risk Assessment Tool. However, only those people who met KINDER 1 Fall Risk Assessment criteria were selected for project inclusion. KINDER 1 category were calculated for comparison. If a participant answered yes to any of the questions, they were selected for project inclusion. If none of the questions were answered with a yes, participants were excluded.
The KINDER 1 Fall Risk Assessment Tool was statistically analyzed, and data was tallied using Poison distribution and placed into quantitative categories that were based upon the binomial risk categories of the KINDER 1 criterion. Of the 3,323 patients who came to the ED during the project period, 328 or 1 in $10(9.9 \%)$ met KINDER 1 criterion and were considered a fall risk. Furthermore, heavy KINDER 1 category made up eighty (80\%) of at-risk patients based on the KINDER 1 criterion. These patients were either over age 70 or had an altered mental status. There was one patient with an altered mental status that fell without injury during project implementation. There was limited free text documentation noted on the KINDER 1 tool during project implementation, therefore this information was omitted from statistical analysis (Figure 5).

Due to the small sample size ( $\mathrm{n}=80)$, the Assesses Care Processes Tools (audit tools) were analyzed for simple statistical comparison using Poisson distribution. The results showed a high established compliance percentage at $97 \%$. This proved that staff implemented all ED fall reduction strategy tools frequently, consistently and accurately. Simple comparative data analysis measured Pre-project and post-project fall rate percentages. This comparative analysis included the previous years (2018) second, third and fourth quarter (ED) patient fall rate percentages, compared to fall rate percentages following project completion. First quarter (2018) ED patient data was not available for use in this project. 


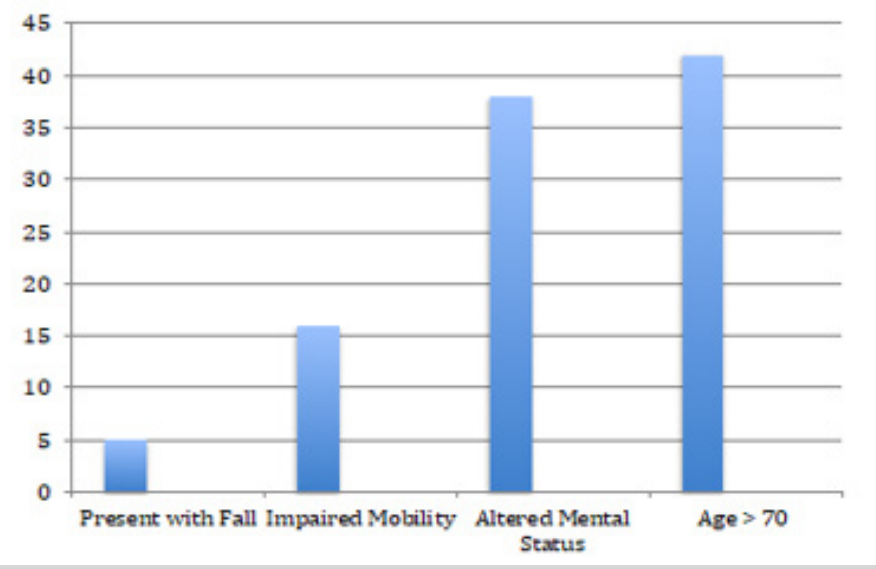

Figure 5: KINDER 1 Fall Risk Category Results.

*These percentages were calculated based upon category.

During the second quarter of 2018, a total of 5,275 patients presented to the ED, and of those patients, three fell and were injured (fall rate $0.70 \%$ ). The findings for the third quarter in 2018, included a total of 6,350 patients that presented to the ED, and of those patients, seven experienced a fall (fall rate 1.10\%) with five patients injured. The results for the fourth quarter of 2018 showed a total of 5,710 patients that presented to the ED, and of those patients, eight experienced a fall (fall rate 1.40\%). Seven patients were injured. Patient falls with injury were not statistically measured or compared for purposes of this project.
During the project intervention period (first quarter of 2019), a total of 3,323 patients presented to the ED, and of those patients, one experienced a fall without injury (fall rate $0.30 \%$ ). The detailed results proved that despite a downward trend in ED patient falls between the fourth quarter of 2018 and completion of the first quarter of 2019, the ED fall rate percentage did not prove to be statistically significant at the 95\% Confidence Interval (CI) using Poisson distribution. This result was attributed to the rather short duration of this study (one quarter) (Figure 6).

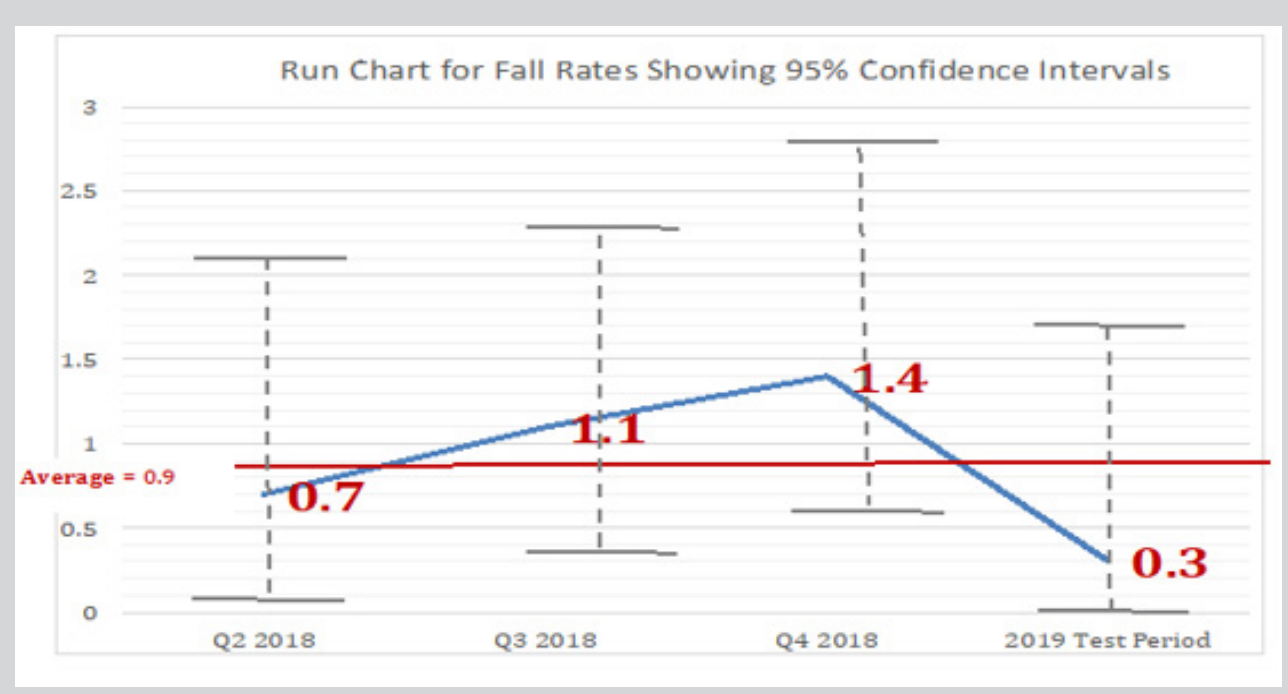

Figure 6: Fall rate run chart.

*Numbers for each category are represented by ED fall rate calculation \%percentage.

Although the KINDER 1 Fall Risk Assessment Tool did not statistically prove to decrease ED patient fall percentages, other benefits of KINDER 1 use were noted. For example, patients at risk for falls were identified very early in the care delivery process. When the KINDER 1 tool was implemented by the triage nurse, early identification of those patients at risk for falls occurred immediately at the point of patient entry. This process directly led to increased patient fall communication among various members of the ED staff, including all Nurses, Physicians, Emergency Medical Technicians and Unit Clerks. An additional benefit of KINDER 1 Tool use included rapid implementation of the AHRQ Schedule Rounding Protocol (standardized hospital falls precautions).

\section{CONCLUSION}

The implementation of a multifactorial, patient-centered approach to fall prevention, including family member involvement, can significantly improve patient quality of life and enhance clinical outcomes. Multifactorial patient fall prevention strategies may also work to promote staff communication, enhance safety in the patient care environment, reduce unnecessary fall related expenditures and assist to flag fall risk patients prior to admittance to other inpatient areas within an organization's mesosystem. These factors may further lead to increased patient and employee satisfaction and the avoidance of possible fall related litigation, Hibbard [10]. 
Despite the establishment of a downward trend, the results of this project did not contribute to statistically significant patient fall reduction percentages in the ED. However, a collaborative effort to reduce patient falls in the ED highlighted the effectiveness of KINDER 1 Fall Risk Assessment Tool. Because the KINDER 1 was implemented at the point of patient entry (triage), it proved to promote staff communication and foster a safe environment by minimizing variables that work to compromise patient care. Furthermore, the results of this project overwhelmingly point to the characteristics, implications and values of creating and implementing a customized, multifactorial patient fall reduction strategy that involves rapid patient fall risk identification $[11,12]$.

Patient fall prevention in the ED is possible and has the potential to achieve beneficial outcomes for both the individual and for the supporting healthcare organization. Knowing the many challenges that healthcare organizations face; a successful patient fall reduction strategy has a vital role to play in constituting effective practice change. More longitudinal ED fall reduction research using AHRQ Patient Fall Prevention Guidelines is necessary. Increasing awareness of this issue among staff, stakeholders, patients and families is essential. Nurse leaders in the ED setting should keenly take note.

\section{REFERENCES}

1. McCarty C, Woehle T, Waring S, Taran A, Kitch L (2018) Implementation of the medfrat to promote quality care and decrease falls in community hospital emergency rooms. J Emerg Nurs 44(3): 280-284.

2. The Joint Commission (2018) High reliability healthcare.
3. France D, Slayton J, Moore S, Domenico H, Matthews J, et al. (2017) A multicomponent fall prevention strategy reduces falls at an academic medical center. The Joint Commission Journal on Quality and Patient Safety 43(9): 460-470.

4. Agency for Healthcare Quality and Research (AHQR) (2018) Patient safety organization program.

5. Liu H, Yong HP, Peck HO, Clark R, Matcher D, et al. (2017) Falls efficacy, postural balance, and risk for falls in older adults with falls-related emergency department visits: Prospective cohort study. BMC Geriatr 17(1): 291.

6. Pua Y, Ong P, Clark R, Matcher D, Lim E (2017) Falls efficacy, postural balance, and risk for falls in older adults with falls-related emergency department visits: Prospective cohort study. Biomed Central Geriatrics 17(1): 291.

7. Goldberg E, McCreedy E, Gettel C, Merchant R (2017) A cross sectional study examining older adult emergency department fall history, post fall treatment and prevention. Rhode Island Medical Journal 12(1): 18-23.

8. Townsend A, Ortiz M, Sansweet T (2016) A successful ED fall risk program using the kinder 1 fall risk assessment tool. J Emerg Nurs 42(6): 492-497.

9. Alexander D, Kinsley T, Waszinski C (2013) Journey to a safe environment: Fall prevention in an emergency department at a level I trauma center. J Emerg Nurs 39(4): 346-352.

10. Hibbard J, Greene J (2013) What the evidence shows about patient activation: Better health outcomes and care expenses, fewer data on costs. Health Aff 32(2): 207-214.

11. Shuey K, Balch C (2014) Fall prevention in high-risk patients. Crit Care Nurs Clin North Am 26(4): 569-580.

12. World Health Organization (WHO) (2018) Falls. 